\title{
ISPY - NaCo Imaging Survey for Planets around Young stars
}

\section{A young companion candidate embedded in the $\mathbf{R} \operatorname{CrA}$ cloud $^{\star, \star \star, \star \star \star}$}

\author{
G. Cugno ${ }^{1}$, S. P. Quanz ${ }^{1, \star \star \star \star}$, R. Launhardt ${ }^{2}$, A. Musso Barcucci ${ }^{2}$, S. S. Brems ${ }^{3}$, A. Cheetham ${ }^{4}$, N. Godoy ${ }^{5,6}$, \\ G. M. Kennedy ${ }^{7,8}$, T. Henning ${ }^{2}$, A. Müller ${ }^{2}$, J. Olofsson ${ }^{5,6}$, F. Pepe ${ }^{4}$, A. Quirrenbach ${ }^{3}$, S. Reffert ${ }^{3}$, E. L. Rickman ${ }^{4}$, \\ and D. Ségransan ${ }^{4}$
}

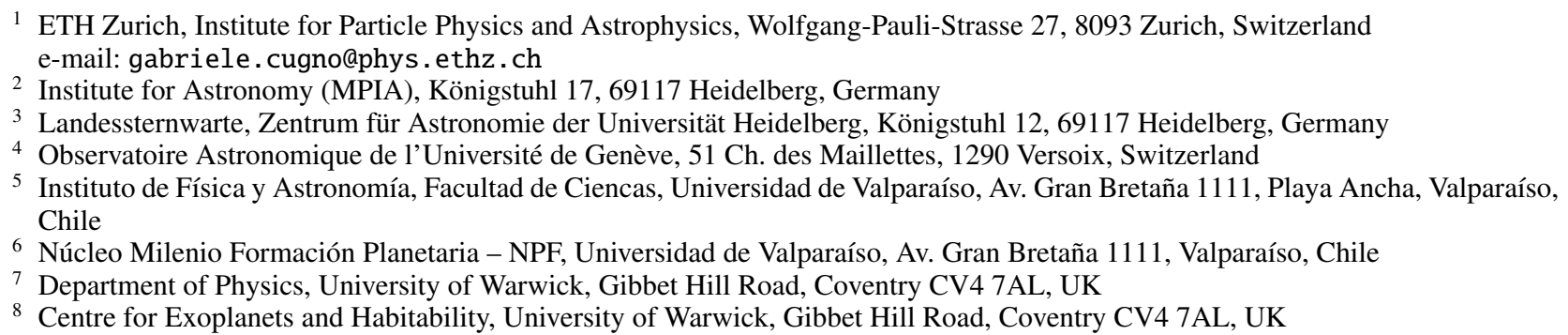

Received 28 January 2019 / Accepted 10 February 2019

\begin{abstract}
Context. Within the NaCo-ISPY exoplanet imaging program, we aim at detecting and characterizing the population of low-mass companions at wide separations $(\gtrsim 10 \mathrm{AU})$, focusing in particular on young stars either hosting a known protoplanetary disk or a debris disk.

Aims. R CrA is one of the youngest (1-3 Myr) and most promising objects in our sample because of two previous studies that suggested the presence of a close companion. Our aim is to directly image and characterize the companion for the first time.

Methods. We observed R CrA twice with the NaCo instrument at the Very Large Telescope (VLT) in the $L^{\prime}$ filter with a one year time baseline in between. The high-contrast imaging data were reduced and analyzed and the companion candidate was detected in both datasets. We used artificial negative signals to determine the position and brightness of the companion and the related uncertainties. Results. The companion is detected at a separation of 196.8 $\pm 4.5 / 196.6 \pm 5.9$ mas $(18.7 \pm 1.3 / 18.7 \pm 1.4 \mathrm{AU})$ and position angle of $134.7 \pm 0.5^{\circ} / 133.7 \pm 0.7^{\circ}$ in the first/second epoch observation. We measure a contrast of $7.29 \pm 0.18 / 6.70 \pm 0.15$ mag with respect to the primary. A study of the stellar proper motion rejects the hypothesis that the signal is a background object. The companion candidate orbits in the clockwise direction and, if on a face-on circular orbit, its period is $\sim 43-47 \mathrm{yr}$. This value disagrees with the estimated orbital motion and therefore a face-on circular orbit may be excluded. Depending on the assumed age, extinction, and brightness of the primary, the stellar companion has a mass between $0.10 \pm 0.02 M_{\odot}$ and $1.03_{-0.18}^{+0.20} M_{\odot}$ range, if no contribution from circumsecondary material is taken into account.

Conclusions. As already hypothesized by previous studies, we directly detected a low-mass stellar companion orbiting the young Herbig Ae/Be star R CrA. Depending on the age assumptions, the companion is among the youngest forming companions imaged to date, and its presence needs to be taken into account when analyzing the complex circumstellar environment of R CrA.
\end{abstract}

Key words. techniques: high angular resolution - planets and satellites: formation - planets and satellites: detection

\section{Introduction}

In recent years, high-contrast high spatial resolution direct imaging has revealed a few planetary mass objects $(\beta$ Pic b, HR 8799 bcde, HD 95086 b, 51 Eri b, HIP 65426 b,

* Based on observations collected at the Paranal Observatory, ESO (Chile). Program ID: 199.C-0065(A) and 1101.C-0092(A).

$\star \star$ After this paper was first submitted, another study of R CrA corroborating the reality of its companion, and reaching conclusions largely in agreement with ours, was posted on arXiv (Mesa et al. 2019).

$\star \star \star$ The data associated with Fig. 1 are only available at the CDS via anonymous ftp to cdsarc.u-strasbg. fr (130.79.128.5) or via http://cdsarc.u-strasbg.fr/viz-bin/qcat?J/A+A/624/A29

$\star \star \star \star$ National Center of Competence in Research "PlanetS" (http:// nccr-planets.ch)
PDS 70 b; Lagrange et al. 2009; Marois et al. 2008, 2010; Rameau et al. 2013; Macintosh et al. 2015; Chauvin et al. 2017b; Keppler et al. 2018). Several surveys (such as NICI, SPHERE-SHINE, IDPS, SEEDS, LEECH, and GPI; Liu et al. 2010; Chauvin et al. 2017a; Vigan et al. 2012; Tamura 2014; Skemer et al. 2014; Macintosh et al. 2014) exploit the capabilities of existing instrumentation to put stronger constraints on the occurrence rate of giant planets $\left(5-13 M_{J}\right)$ at large orbital distance (30-300 AU), which was estimated to be $0.6_{-0.5}^{+0.7} \%$ by Bowler (2016). Statistically characterizing the giant planet population in wide orbits ( $>10 \mathrm{AU})$ is also one of the primary goals of the NaCo Imaging Survey for Planets around Young stars (NaCo-ISPY; Launhardt et al., in prep.). The survey targets nearby young stars that are surrounded by either a protoplanetary or a debris disk. Unlike most other ongoing imaging 
Table 1. Stellar parameters of R CrA.

\begin{tabular}{llc}
\hline \hline Parameter & Values & Reference \\
\hline RA & $19^{\mathrm{h}} 01^{\mathrm{m}} 53.68^{\mathrm{s}}$ & $(1)$ \\
Dec & $-36^{\circ} 57^{\prime} 08.14^{\prime \prime}$ & $(1)$ \\
Sp. type & $\mathrm{F} 5, \mathrm{~A} 5, \mathrm{~B} 8$ & $(2),(3),(4)$ \\
Dist. $(\mathrm{pc})$ & $94.9 \pm 6.3$ & $(1)$ \\
$\mu_{\alpha} \times \cos (\delta)\left(\mathrm{mas} \mathrm{yr}^{-1}\right)$ & $1.58 \pm 1.20$ & $(1)$ \\
$\mu_{\delta}\left(\mathrm{mas} \mathrm{yr}^{-1}\right)$ & $-30.83 \pm 1.19$ & $(1)$ \\
$K(\mathrm{mag})$ & $2.858 \pm 0.262$ & $(5)$ \\
$L(\mathrm{mag})$ & $1.78 \pm 0.05$ & $(6)$ \\
WISE $W 3(\mathrm{mag})$ & $-1.239 \pm 0.390$ & $(7)$ \\
Age $(\mathrm{Myr})$ & $0.3-3,1$ & $(8),(9),(10)$ \\
$A_{V}(\mathrm{mag})$ & $6.0-8.0,1.9$ & $(9),(11)$ \\
Mass $\left(M_{\odot}\right)$ & $3.0,3.5$ & $(10),(11)$ \\
\hline
\end{tabular}

References. (1) Gaia Collaboration (2018), (2) Hillenbrand et al. (1992), (3) Chen et al. (1997), (4) Hamaguchi et al. (2005), (5) Cutri et al. (2003), (6) Glass \& Penston (1975), (7) Cutri et al. (2013), (8) Meyer \& Wilking (2009), (9) Forbrich et al. (2006), (10) Bibo et al (1992), (11) Lorenzetti et al. (1999).

surveys that focus on shorter wavelengths, ISPY utilizes the $L^{\prime}$ filter $(\lambda \sim 3.8 \mu \mathrm{m})$ of the $\mathrm{NaCo}$ instrument at the Very Large Telescope (VLT) to obtain high-contrast imaging data. At this wavelength, there are four main advantages compared to the near-infrared regime: (1) the contrast requirements are more favorable for a detection; (2) dust extinction from circumstellar material has minimal effects on the observed flux; (3) scattering from circumstellar disk material, which increases the noise and probability of false positives, is less important; and (4) contamination from background objects is less severe because most background stars are not red enough to be easily detected at $L^{\prime}$ band. Altogether, possibly embedded objects with lower intrinsic luminosity might be detected. The major limiting factor of collecting data in the $3-5 \mu \mathrm{m}$ range is related to the lower spatial resolution that can be achieved.

Given its age and proximity (see Table 1), R CrA is an ideal target for the ISPY search for companions at small physical separations. R CrA is located in the Coronae Australis molecular cloud, a star-forming region containing several low- to intermediate-mass objects (e.g., Fernández \& Comerón 2001; Haas et al. 2008). Its "Coronet" (Taylor \& Storey 1984; Wilking et al. 1997) is a deeply embedded cluster of young stars that was extensively studied in recent years across all wavelengths, from radio (e.g., Brown 1987; Feigelson et al. 1998; Forbrich et al. 2006) to X-rays (e.g., Koyama et al. 1996; Neuhäuser et al. 2000), and whose luminosity is dominated by R CrA $\left(L_{\mathrm{bol}}=70 L_{\odot}\right.$, Lorenzetti et al. 1999, but updated with distance from Table 1). Because of the complexity of its circumstellar environment and its known variability, the properties of $\mathrm{R} \mathrm{CrA} \mathrm{are} \mathrm{not} \mathrm{well} \mathrm{constrained} \mathrm{(see} \mathrm{Table} \mathrm{1).}$

As an example, the extinction in the R CrA cloud is highly uncertain, and X-ray spectra from Forbrich et al. (2006) suggested $A_{V}=6.0-8.0 \mathrm{mag}$, but other studies derived lower values (e.g., Lorenzetti et al. 1999, $A_{V}=1.9 \mathrm{mag}$ ). Since it was not possible to further constrain this parameter from the available data, we adopted a conservative approach considering $A_{V}$ in the 1.9-8.0 mag range. Concerning possible companions, Takami et al. (2003) observed R CrA with the Royal Greenwich Observatory (RGO) spectrograph on the Anglo-Australian Telescope (AAT) and the data showed a displacement around the $\mathrm{H} \alpha$ line emission that was best explained with the presence of a companion and an outflow from the primary. Combining their
Table 2. Summary of observations.

\begin{tabular}{|c|c|c|}
\hline Parameter & Epoch 1 & Epoch 2 \\
\hline UT date (yyyy/mm/dd) & $2017 / 05 / 19$ & $2018 / 06 / 06$ \\
\hline Prog. ID & 199.C-0065(A) & 1101.C-0092(A) \\
\hline $\operatorname{DIT}^{a}(\mathrm{~s})$ & 0.35 & 0.1082 \\
\hline \# of DITs & 12100 & 18401 \\
\hline Par. angle start/end $\left(^{\circ}\right)$ & $42.46 / 78.44$ & $-40.25 / 80.91$ \\
\hline Airmass $\min / \max$ & $1.04 / 1.19$ & $1.04 / 1.22$ \\
\hline Mean seeing ${ }^{b}$ (as) & 0.65 & 0.63 \\
\hline True north ${ }^{c}\left({ }^{\circ}\right)$ & $0.39 \pm 0.21$ & $0.43 \pm 0.18$ \\
\hline Plate Scale (mas pix ${ }^{-1}$ ) & $27.21 \pm 0.10$ & $27.21 \pm 0.08$ \\
\hline \# unsaturated PSF & 2000 & 2400 \\
\hline DIT unsaturated PSF (s) & 0.005 & 0.004256 \\
\hline
\end{tabular}

Notes. ${ }^{(a)}$ DIT $=$ Detector integration time, i.e., exposure time per image frame. ${ }^{(b)}$ Mean DIMM seeing measured during the observations. ${ }^{(c)}$ Additional rotation in clockwise direction that has to be applied in order for north to point to the top of the images.

results with previous observations presented in Bailey (1998) and Takami et al. (2003) gave a rough estimate for the position angle (PA) of a possible companion in 1996 and 1999 $\left(\sim 220^{\circ}\right.$ and $\left.\sim 200^{\circ}\right)$. Later, Forbrich et al. (2006) hypothesized that a companion was orbiting R CrA based on Chandra and $X M M$-Newton data. These authors detected variable $\mathrm{X}$-ray emission but because no X-ray flux is expected from intermediatemass young stellar objects, they suggested that the emission comes from a low-mass companion. Kraus et al. (2009) analyzed VLTI/AMBER $H$ - and $K$-band spectro-interferometric observations of R CrA and found no clear evidence for a companion at separations between 0.02 and $0 . ' 2$, despite strong asymmetries in the brightness distribution of the source that could be caused by a second object. They achieved contrast limits of 4.0 mag, so a fainter (i.e., lower mass or more deeply embedded) object would have been missed in their observations. Furthermore, they measured the inclination of the disk surrounding R CrA to be $\sim 35^{\circ}$, (confirming previous results from, e.g., Clark et al. 2000) and its PA to be $\sim 180^{\circ}-190^{\circ}$.

In this paper, we present the results of new high-contrast imaging $L^{\prime}$-band observations of R CrA that find direct evidence for a stellar companion. In Sect. 2 we summarize the observations and explain the data reduction. In Sect. 3 we present our results and discuss them in Sect. 4. We conclude in Sect. 5.

\section{Observations and data reduction}

\subsection{VLT/NaCo observations}

We observed R CrA on 2017 May 19 and 2018 June 06, taking advantage of the AGPM vortex vector coronagraph (Mawet et al. 2013), which enables high-contrast imaging very close to the diffraction limit ( $\left.\sim 0^{\prime} 095\right)$. For the observations, the L27 camera (pixel scale $\sim 27.2$ mas pix $\left.^{-1}\right)$ was used with the $L^{\prime}$ filter $\left(\lambda_{c}=\right.$ $3.8 \mu \mathrm{m}, \Delta \lambda=0.62 \mu \mathrm{m})$. The camera derotator was switched off to enable angular differential imaging (ADI; Marois et al. 2006) and the "cube mode" allowed us to save all individual exposures. Both observing runs consisted of series of 13 coronagraphic cubes of images of R CrA (7.6 and 2.3 min for the two epochs) interposed with one cube of sky images to sample the background and subtract it later. In total, for the first and second observations we obtained 70.6/33.2 min of on-source integration time and $36.0 / 121.2^{\circ}$ of field rotation (see Table 2 ). In addition, unsaturated photometric images were taken at the beginning and 
at the end of the observations. These were used later to calibrate, simulate, and insert artificial point spread functions (PSFs) in the data. Table 2 summarizes the observations together with the atmospheric conditions.

\subsection{Data reduction}

Both datasets were reduced with PynPoint (Amara \& Quanz 2012; Stolker et al. 2019), an end-to-end modular python pipeline for analyzing high-contrast imaging data. In particular, the PSF subtraction is based on principal component analysis (PCA; Amara \& Quanz 2012; Soummer et al. 2012).

After assigning to each frame its parallactic angle by linearly interpolating between the start and end value of each cube, the first ten frames and the last frame of each cube were removed. The first frames suffer from a systematically higher background that drops exponentially (e.g., Stolker et al. 2019). The last frame represents the mean of the cube. The remaining frames were dark-subtracted, and the stripes present in the lower left quadrant due to detector issues were corrected by substituting each pixel value with the mean of the two neighboring values. For the second epoch, where two neighboring bad working columns were present on the detector, the average of the closest functioning right and left pixels were used ${ }^{1}$. Other bad pixels were corrected by 4-sigma clipping (Stolker et al. 2019). Then, using spline interpolation all images were aligned applying cross-correlation and the entire stack was shifted to the exact image center after fitting a 2D Gaussian to the mean of the frames. Next, each background cube was averaged to a single image. From each science frame, the average between the previous and subsequent background image was subtracted. Finally, we binned, i.e., mean-combined, sets of two, five, and ten frames. In this way, three different datasets were created and a frame selection was applied to each of the three stacks rejecting images, where the stellar flux measured in a circular aperture $\left(r=1^{\prime \prime}\right.$. 0$)$ deviated by more than three standard deviations from the median values of the stack.

For the PSF subtraction, PynPoint applies full-frame PCA to reconstruct and then remove the stellar PSF before derotating and combining all the images (Amara \& Quanz 2012; Stolker et al. 2019). In order to search for faint high-contrast signals at small separations, several mask radii are applied to cover the central part of the stellar PSF and the number of principal components (PC) used to model and subtract the PSF was varied.

The unsaturated PSF frames underwent a simpler calibration. After dark subtraction and bad pixel cleaning, the background was subtracted using the dithering technique, in which the star is imaged in three different quadrants of the detector ${ }^{2}$. Whenever the star was not present in a quadrant, these cubes served to sample the background. Because the dither position changed every 400 frames, the images were subtracted from each other with an offset of 400 frames, meaning that the $n$th image of the set was subtracted from the $(n+400)$ th frame and cut $(0.5$ in size $)$ around the positive PSF signal. Then, all frames were aligned, centered, and averaged to obtain a unique PSF template for each epoch.

We looked for differences in the unsaturated images within individual epochs and between them. Since the final PSF template is unique for each dataset, we have to consider variations

\footnotetext{
1 We note, however, that the stripe correction has no influence on the results presented later since the studied region was located in a different quadrant.

2 We avoided using the quadrant with bad columns.
}

of the unsaturated images due to changes in weather conditions and possibly in intrinsic stellar flux. This component of the photometric uncertainty was estimated as the standard deviation of the stellar flux measured in each exposure in a circular aperture of $0.19(2 \lambda / D)$ and it amounts to 0.03 mag for epoch 1 and $0.09 \mathrm{mag}$ for epoch 2 . The larger variation in epoch 2 results from a systematic decrease in stellar flux by $\sim 13 \%$ between the beginning and end of the observing sequence. Furthermore, during the first epoch, the central star R CrA appeared on average 0.22 mag brighter than during the second observation ${ }^{3}$. This difference is analyzed in the context of the companion brightness in Sect. 4.

\section{Analysis and results}

\subsection{The detection of a young companion candidate}

A strong companion signal is consistently detected in both datasets (see Fig. 1) and, given the baseline of one year, the displacement is in agreement with orbital motion as we discuss in Sect. 4. The companion candidate is detected for a wide range in the number of subtracted PCs (1-20 for Epoch 1 and 4-30 for Epoch 2) applying inner masks of $0 . ' 05$ and $0 .^{\prime} 1$ in radius. To quantify the confidence of the detection, we calculated the false positive fraction (FPF) measuring the signal flux in an aperture of diameter $\lambda / D\left(\approx 0{ }^{\prime} 095\right)$ and sampling the residual noise in non-overlapping apertures of the same size at the same separation, but at different azimuthal angles (Mawet et al. 2014). The central position of the signal aperture was determined by fitting a 2D Gaussian to the signal. Using Eqs. (9) and (10) of Mawet et al. (2014) we determined the signal-to-noise ratio $(\mathrm{S} / \mathrm{N})$ and the FPF. This approach considers corrections for small sample statistics and utilizes the Student t-distribution to estimate FPFs. We found a best $\mathrm{S} / \mathrm{N}$ of 9.2 for the first epoch (no frame binning, $\mathrm{PC}=5, r_{\text {mask }}=0$. $^{\prime} 05$ as) and 9.6 for the second (bin $=10, \mathrm{PC}=9, r_{\text {mask }}=0.1$ ), corresponding to FPFs of $1.7 \times 10^{-6}$ and $1.17 \times 10^{-6}$, respectively.

\subsection{Astrometry and photometry}

To determine the astrometry and photometry of the companion, two different methods were used, namely the minimization of the determinants of the Hessian matrix (HM) at the position of the signal (Cugno et al. 2019) and a Markov chain Monte Carlo (MCMC) minimization algorithm (Stolker et al. 2019). Both methods are based on the artificial planet technique, in which signals from unsaturated PSFs are subtracted from the data with different combinations of position and contrast to minimize the residuals. Specifically, the HM technique tries to minimize the curvature of the image in the signal region and the MCMC minimizes the flux residuals as performed in Wertz et al. (2017). In both cases a circular region with $r=0{ }^{\prime} 1425(1.5 \lambda / D)$ around the companion was considered.

We used the HM characterization algorithm on all combinations of PCs and the mask radius that yielded an FPF $<3.2 \times 10^{-5}$ (corresponding to a $4 \sigma$ confidence) in the bin $=5$ and bin $=$ 10 datasets (22 setups for epoch 1 and 56 for epoch 2). To consider systematic effects related to the PSF subtraction parameters, we averaged the best fit solutions to obtain astrometry and photometry of the companion. The uncertainties are represented by the standard deviation of the set of best solutions.

\footnotetext{
3 This value is based on the measured count rate, even though the conditions were not photometric either night.
} 
Original image
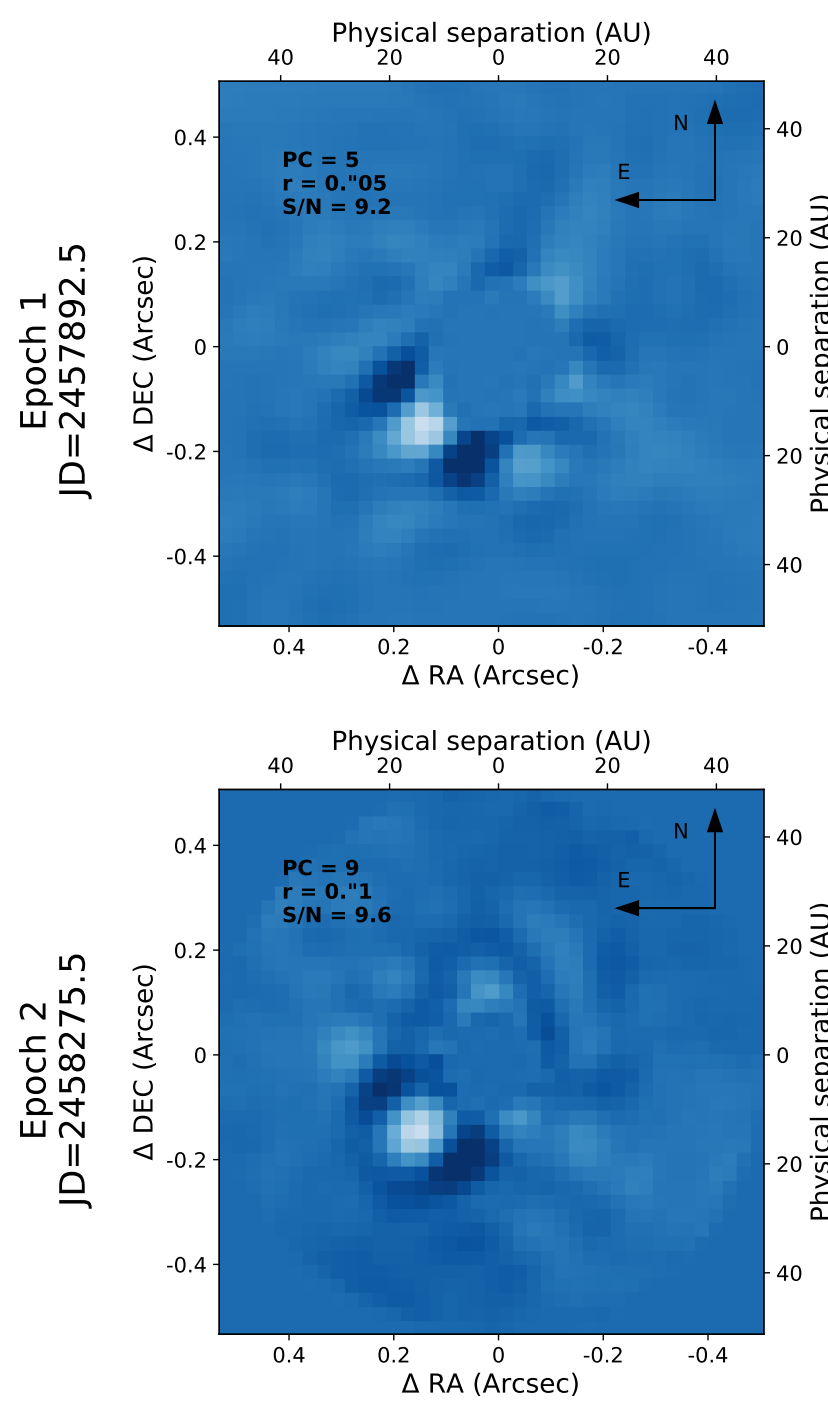

Signal subtracted
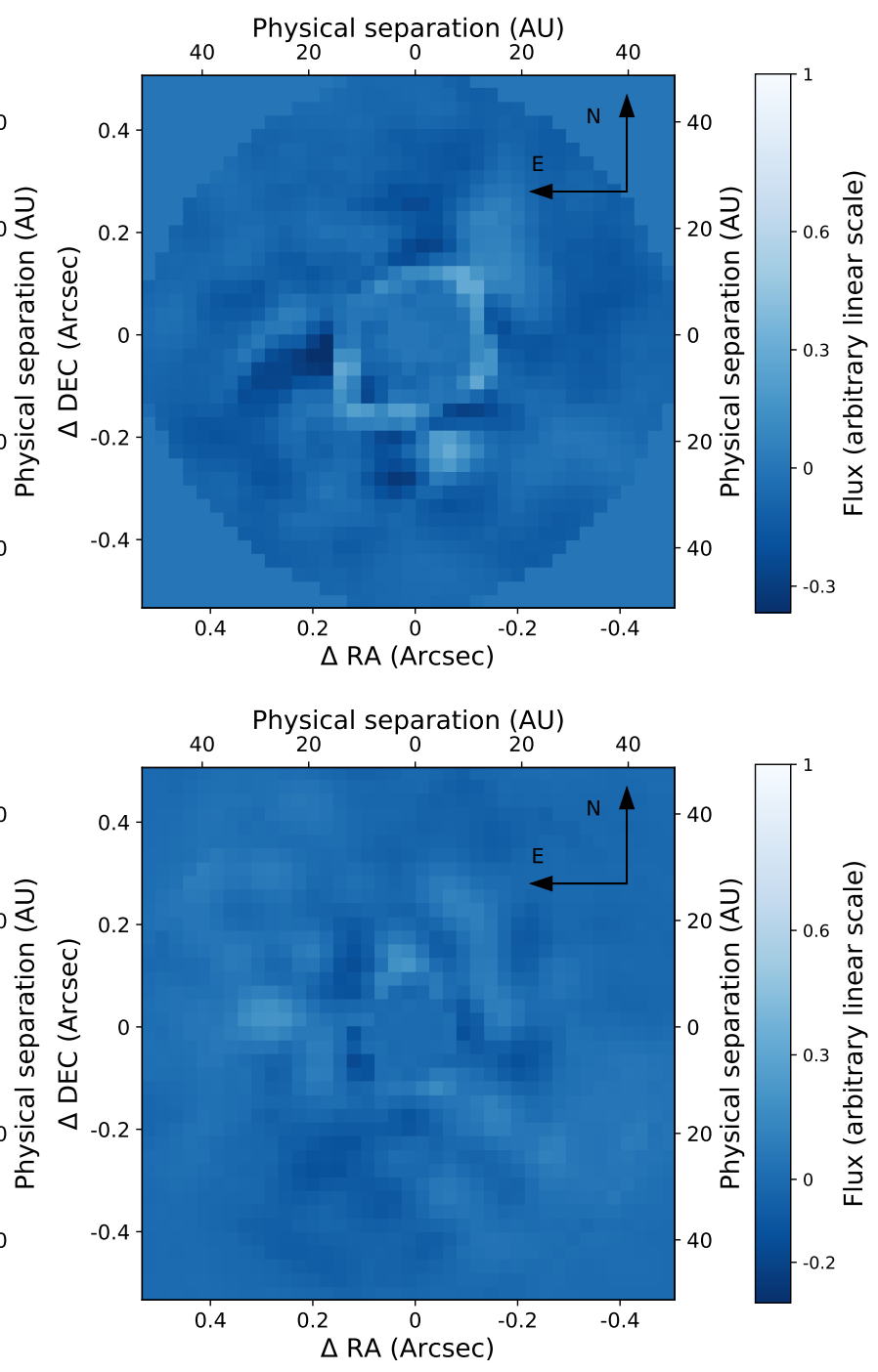

Fig. 1. Left panel: first and second epoch detections (top and bottom panels, respectively) with the highest S/N (left panel) and the corresponding signal-subtracted images (right panel). The numbers of subtracted PCs from the central PSF, the applied mask radius, and the $\mathrm{S} / \mathrm{N}$ are reported in the top left corner of the original images. The same parameters were used for the right panels, where prior to PSF subtraction the HM approach was used to subtract the companion signal.

Table 3. Measured astrometry and photometry of R CrA B.

\begin{tabular}{llll}
\hline \hline Epoch 1 & Sep (mas) & PA $\left({ }^{\circ}\right)$ & Contrast (mag) \\
\hline Hessian matrix & $196.8 \pm 4.5$ & $134.7 \pm 0.5$ & $7.29 \pm 0.18$ \\
MCMC & $192.9 \pm 1.0$ & $135.0 \pm 0.2$ & $6.93_{-0.02}^{+0.01}$ \\
\hline Epoch 2 & & & \\
\hline Hessian matrix & $196.6 \pm 5.9$ & $133.7 \pm 0.7$ & $6.70 \pm 0.15$ \\
MCMC & $186.7 \pm 1.1$ & $132.0 \pm 0.2$ & $6.48 \pm 0.01$ \\
\hline
\end{tabular}

The MCMC characterization algorithm was performed for stacks with 10 binned images using the parameter combination that delivered the lowest FPF, namely PC $=3$, mask $=0$.'05 for epoch 1 , and $\mathrm{PC}=9$, mask $=0$ '! 1 for epoch 2 . The parameter space was explored by 200 walkers, each performing a chain of 500 steps.

Astrometric calibration was performed using an astrometric reference field in 47 Tuc taken on 2017 May 19 and on 2018
June 07 and comparing it to the catalog of McLaughlin et al. (2006). The resulting True North correction and platescale are summarized in Table 2. The measured contrasts were corrected for the separation-dependent throughput of the coronagraph (Mawet et al. 2013), which was 0.91 for both epochs. Table 3 summarizes the measurements of the companion for both methods and epochs after those corrections, still without considering photometric variability. The formal errors obtained through the MCMC algorithm do not include any systematic uncertainty and therefore are much smaller than those obtained for HM. For epoch 1, the astrometric results obtained through both methods agree within $1 \sigma$ error bars, while the measurements obtained for the data of epoch 2 show a larger scatter. For both cases, the MCMC approach measured a lower contrast than the HM. To investigate the presence of systematics, we inserted signals at 0.2 at three different PAs randomly chosen (but separated by at least $50^{\circ}$ from the companion) with 7.3 mag contrast. The analysis shows that neither of the two approaches systematically delivers more accurate results; sometimes the HM algorithm 


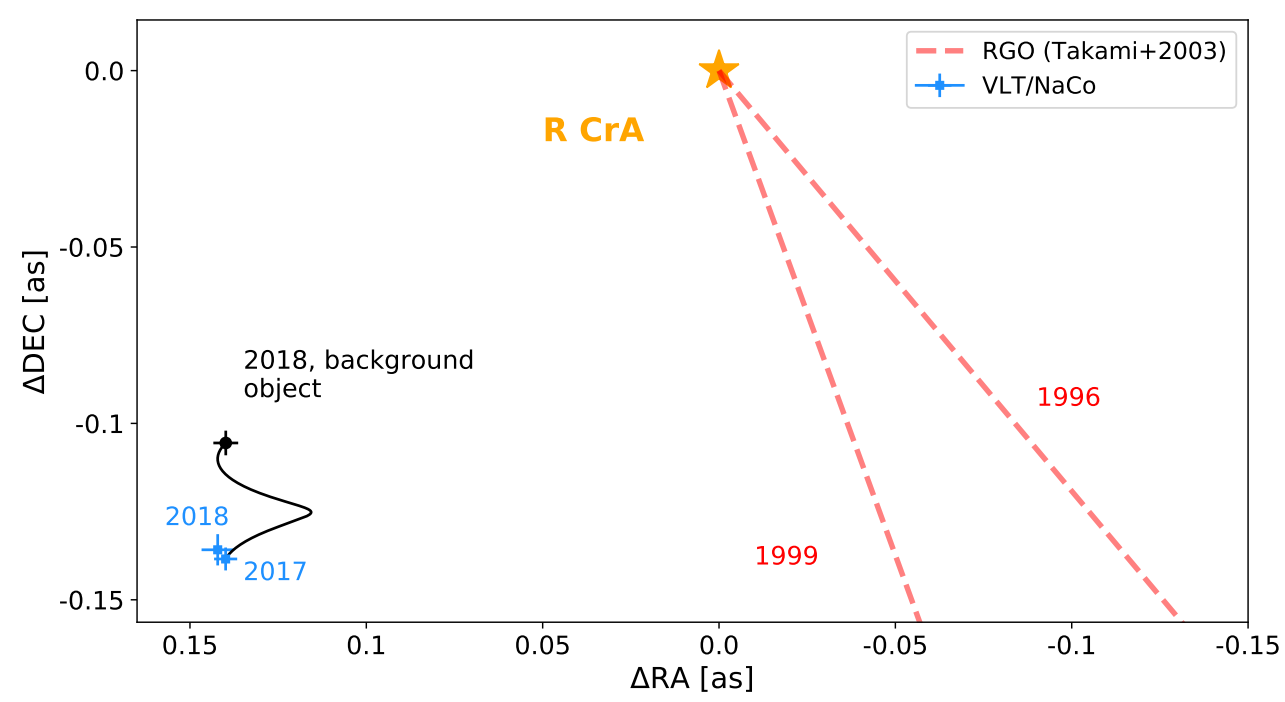

Fig. 2. Astrometry chart of R CrA and its companion candidate. The orange asterisk represents the position of $\mathrm{R} \mathrm{CrA \text {, }}$ the blue squares represent the detected relative positions presented in this work, while the black circle shows the expected position of a stationary background object at the second epoch, given the parallax and proper motion of $\mathrm{R} C r A$ as reported in Table 1. The red dashed lines represent the PAs at which the companion was predicted by Takami et al. (2003) from 1996 and 1999 data. gives values closer to the known ground-truth, sometimes the MCMC sampling. This is most probably because of the presence of residual PSF speckle noise in the image that affects the measurements. Because the HM approach includes at least in part systematic uncertainties, we decided to use these results for the remainder of our analysis.

\section{Discussion}

\subsection{Exclusion of a background source}

In Fig. 2 we show in blue squares the relative astrometric position of $\mathrm{RCrA} \mathrm{B}$, keeping the primary at a fixed position. The black line and circle represent the path and position we would expect for a stationary background object according to the Gaia proper motion of R CrA, which is inconsistent with the observations. Error bars represent uncertainties for the astrometric results obtained with the HM approach and also include the uncertainties in Gaia parallax and proper motion for the black circle. However, both the Gaia DR2 astrometric excess noise of 3.3 mas and the large unit weight error of $\approx 31$ (UWE; the currently most useful goodness-of-fit indicator for the Gaia DR2 five-parameter astrometric solutions; Lindegren et al. 2018) indicate that the single star astrometric solution does not fit the observations well. These inconsistencies could be due to systematic errors not taken into account by the formal uncertainties, but at the given level it is more likely that the companion to $\mathrm{R} \mathrm{CrA}$ is responsible for the larger than expected residuals to the single star solution.

Combining predictions from Takami et al. (2003) with the new information from $\mathrm{NaCo}$ observations, it seems that the secondary source moves west to east and, if bound, it orbits $\mathrm{RCrA}$ in a clockwise direction (current projected separation $\sim 18.7 \mathrm{AU})$. Assuming a face-on circular orbit, its period would be $\sim 43-47 \mathrm{yr}$ (central star mass $\sim 3.0-3.5 M_{\odot}$ ). This value

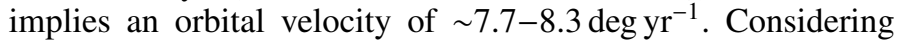
the PAs estimated between 1996 and 2018, the orbital velocity was calculated to be $\sim 4.0 \mathrm{deg} \mathrm{yr}^{-1}$, while that between the ISPY detections is $1 \pm 0.9 \mathrm{deg} \mathrm{\textrm {yr } ^ { - 1 }}$. Therefore, based on the 22 -yearlong baseline, the inconsistency allows us to marginally exclude a face-on orbit. Given the lack of uncertainties, confidence levels, and orbital separations in the predictions from Takami et al. (2003), it is not possible to draw more significant conclusions and more precise constraints from the available information.

\subsection{Mass of the companion candidate}

To estimate the $L^{\prime}$ brightness of RCrA, we considered the 2MASS (Cutri et al. 2003) $K$-band photometry and that from the AllWISE Catalog (Cutri et al. 2013) in $W 3(\lambda \sim 11.6 \mu \mathrm{m})$ as reported in Table 1 . We estimated fluxes in the two bands using filter zero points ${ }^{4}$ and interpolated between the two values. The resulting flux was translated into magnitude and we obtained a brightness of $1.5 \pm 0.5 \mathrm{mag}$ at $3.8 \mu \mathrm{m}$. This method avoids using the highly saturated and therefore rather unreliable measurements taken with the WISE (Wright et al. 2010) $W 1$ and $W 2$ bands. This estimate agrees with the brightness measured by Glass \& Penston (1975), which is $1.78 \pm 0.05 \mathrm{mag}$, where the error is an upper limit for the uncertainty. To be conservative, we considered $L^{\prime}$ values for the $\mathrm{R} \mathrm{CrA}$ system to be in the 2.0-1.0 mag range. It is likely that the measured infrared emission is affected by contribution from the circumstellar disk and surrounding material. Nevertheless, the contrast has been measured with respect to the observed flux, and the intrinsic stellar brightness is not relevant for the characterization of the companion. After combining the primary brightness with the measured contrasts, we corrected for extinction $\left(A_{V}=1.9-8.0 \mathrm{mag}\right.$, Table 1) using the extinction curve from Mathis (1990) for $R_{V}=$ 3.1. This resulted in $A_{L^{\prime}}=0.09-0.36 \mathrm{mag}$. Afterward, absolute magnitudes for the companion candidate were computed and compared with the interpolated isochrones from the BT-Settl models (Baraffe et al. 2003) for ages of $\tau=1,2$, and 3 Myr. As shown in Fig. 3, depending on the brightness, extinction, and age of the primary at the time of the observations, the companion could have masses ranging from $0.10 \pm 0.02 M_{\odot}$ to $1.03_{-0.18}^{+0.20} M_{\odot}$. Considering the particular case in which the brightness of the primary was equal to $1.78 \pm 0.05 \mathrm{mag}$, as measured by Glass \& Penston (1975) at $3.5 \mu \mathrm{m}$, the companion would have a mass of $0.10-0.38 M_{\odot}$ (epoch 1) and $0.19-0.63 M_{\odot}$ (epoch 2) for ages in the 1-3 Myr range and $L^{\prime}$ extinctions in the aforementioned interval. These ranges include $1 \sigma$ error bars.

As calculated in Sect. 2, R CrA appeared $\sim 0.22$ mag brighter in the first observation than in the second. Even if this offset were due to intrinsic stellar variation, the estimated contrasts (Table 3) indicate that the $L^{\prime}$ brightness of the companion is subject to variability of $\Delta$ mag $\approx 0.4 \pm 0.23$ mag, making the mass estimation through model grids particularly difficult. Variability

\footnotetext{
4 http://svo2.cab.inta-csic.es/theory/fps3/
} 

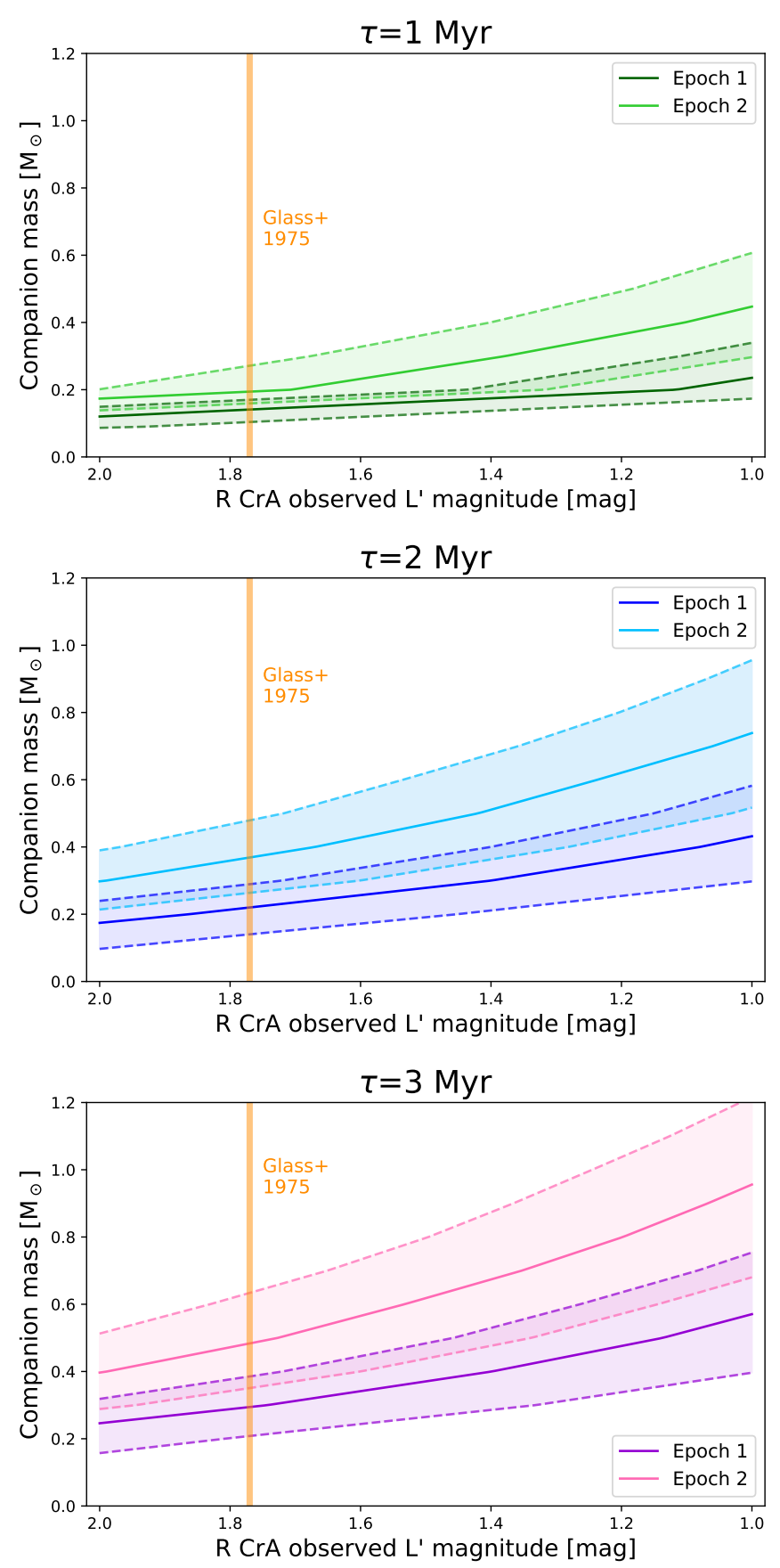

Fig. 3. Companion mass as a function of the primary $L^{\prime}$ magnitude for $\tau=1,2,3 \mathrm{Myr}$ (top to bottom panels). The shaded regions represent the $1 \sigma$ uncertainty range coming from contrast, photometric uncertainty of the PSF template, distance, and extinction. The orange solid line indicates the value provided by Glass \& Penston (1975).

of this order of magnitude is expected for low-mass young protostars (e.g., Cody \& Hillenbrand 2014), in particular if part of the flux is due to accretion processes occurring at varying rates (Wolk et al. 2013) and emitting infrared flux. Another process that might contribute to the observed $L^{\prime}$ flux is the reprocessing of stellar photons that are absorbed by circumstellar material and re-emitted in the mid-infrared. Unfortunately, with the available data, it is not possible to estimate the fraction of reprocessed $L^{\prime}$ flux. Accounting for the presence of accreting circumstellar material and a dusty envelope, the estimated mass of the companion should be considered an upper limit.

\section{Summary and conclusions}

As shown in several previous studies, the environment in the Coronet is extremely complex. Our $\mathrm{NaCo}$ high-contrast imaging data revealed an additional physical component of this region, which will need to be taken into account when interpreting the cloud environment harboring $\mathrm{R} \mathrm{CrA}$. Follow-up observations with a baseline of a few years will allow to better constrain the orbit of the object and further investigate its time variability. High-contrast observations at shorter wavelengths may shed light on the spectral type of the object and help quantify extinction effects. Finally, the presence of material accreting onto the companion candidate and emitting infrared flux needs to be considered and investigated. In this case, different contributions to the infrared emission should be disentangled and the object mass could be calculated from the true photospheric emission of the object.

Acknowledgements. The authors thank T. Stolker and C. Bailer-Jones for helpful and constructive discussions. Part of this work has been carried out within the frame of the National Center for Competence in Research PlanetS supported by the Swiss National Science Foundation. SPQ acknowledges the financial support of the SNSF. GC and SPQ thank the Swiss National Science Foundation for financial support under grant number 200021_169131. GMK is supported by the Royal Society as a Royal Society University Research Fellow. AM acknowledges the support of the DFG priority program SPP 1992 "Exploring the Diversity of Extrasolar Planets" (MU 4172/1-1). JO and NG acknowledge financial support from the ICM (Iniciativa Científica Milenio) via the Núcleo Milenio de Formación Planetaria grant. JO acknowledges support from the Universidad de Valparaíso, and from Fondecyt (grant 1180395). This research has made use of the SIMBAD database, operated at CDS, Strasbourg, France. This work has made use of data from the European Space Agency (ESA) mission Gaia (https://www.cosmos.esa.int/gaia), processed by the Gaia Data Processing and Analysis Consortium (DPAC, https://www.cosmos.esa.int/ web/gaia/dpac/consortium). Funding for the DPAC has been provided by national institutions, in particular the institutions participating in the Gaia Multilateral Agreement. This publication makes use of data products from the Widefield Infrared Survey Explorer, which is a joint project of the University of California, Los Angeles, and the Jet Propulsion Laboratory/California Institute of Technology, funded by the National Aeronautics and Space Administration.

\section{References}

Amara, A., \& Quanz, S. P. 2012, MNRAS, 427, 948

Bailey, J. 1998, MNRAS, 301, 161

Baraffe, I., Chabrier, G., Barman, T. S., Allard, F., \& Hauschildt, P. H. 2003, A\&A, 402, 701

Bibo, E. A., The, P. S., \& Dawanas, D. N. 1992, A\&A, 260, 293

Bowler, B. P. 2016, PASP, 128, 102001

Brown, A. 1987, ApJ, 322, L31

Chauvin, G., Desidera, S., Lagrange, A. M., et al. 2017a, in SF2A-2017. Proceedings of the Annual meeting of the French Society of Astronomy and Astrophysics, eds. C. Reylé, P. Di Matteo, F. Herpin, et al., 331

Chauvin, G., Desidera, S., Lagrange, A.-M., et al. 2017b, A\&A, 605, L9

Chen, H., Grenfell, T. G., Myers, P. C., \& Hughes, J. D. 1997, ApJ, 478, 295

Clark, S., McCall, A., Chrysostomou, A., et al. 2000, MNRAS, 319, 337

Cody, A. M., \& Hillenbrand, L. A. 2014, ApJ, 796, 129

Cugno, G., Quanz, S. P., Hunziker, S., et al. 2019, A\&A, 622, A156

Cutri, R. M., Skrutskie, M. F., van Dyk, S., et al. 2003, VizieR Online Data Catalog: II/246

Cutri, R. M., Wright, E. L., Conrow, T., et al. 2013, VizieR Online Data Catalog: II/328

Feigelson, E. D., Carkner, L., \& Wilking, B. A. 1998, ApJ, 494, L215

Fernández, M., \& Comerón, F. 2001, A\&A, 380, 264

Forbrich, J., Preibisch, T., \& Menten, K. M. 2006, A\&A, 446, 155

Gaia Collaboration (Brown, A. G. A., et al.) 2018, A\&A, 616, A1

Glass, I. S., \& Penston, M. V. 1975, MNRAS, 172, 227

Haas, M., Heymann, F., Domke, I., et al. 2008, A\&A, 488, 987

Hamaguchi, K., Yamauchi, S., \& Koyama, K. 2005, ApJ, 618, 360

Hillenbrand, L. A., Strom, S. E., Vrba, F. J., \& Keene, J. 1992, ApJ, 397, 613

Keppler, M., Benisty, M., Müller, A., et al. 2018, A\&A, 617, A44

Koyama, K., Hamaguchi, K., Ueno, S., Kobayashi, N., \& Feigelson, E. D. 1996, PASJ, 48, L87 
G. Cugno et al.: A young companion candidate embedded in the R CrA cloud

Kraus, S., Hofmann, K.-H., Malbet, F., et al. 2009, A\&A, 508, 787

Lagrange, A.-M., Gratadour, D., Chauvin, G., et al. 2009, A\&A, 493, L21

Lindegren, L., Hernández, J., Bombrun, A., et al. 2018, A\&A, 616, A2

Liu, M. C., Wahhaj, Z., Biller, B. A., et al. 2010, in Adaptive Optics Systems II, Proc. SPIE, 7736, 77361K

Lorenzetti, D., Tommasi, E., Giannini, T., et al. 1999, A\&A, 346, 604

Macintosh, B., Graham, J. R., Ingraham, P., et al. 2014, Proc. Natl. Acad. Sci., 111,12661

Macintosh, B., Graham, J. R., Barman, T., et al. 2015, Science, 350, 64

Marois, C., Lafrenière, D., Doyon, R., Macintosh, B., \& Nadeau, D. 2006, ApJ, 641, 556

Marois, C., Macintosh, B., Barman, T., et al. 2008, Science, 322, 1348

Marois, C., Zuckerman, B., Konopacky, Q. M., Macintosh, B., \& Barman, T. 2010, Nature, 468, 1080

Mathis, J. S. 1990, ARA\&A, 28, 37

Mawet, D., Absil, O., Delacroix, C., et al. 2013, A\&A, 552, L13

Mawet, D., Milli, J., Wahhaj, Z., et al. 2014, ApJ, 792, 97

McLaughlin, D. E., Anderson, J., Meylan, G., et al. 2006, ApJS, 166, 249

Mesa, D., Bonnefoy, M., Gratton, R., et al. 2019, A\&A, 624, A4
Meyer, M. R., \& Wilking, B. A. 2009, PASP, 121, 350

Neuhäuser, R., Walter, F. M., Covino, E., et al. 2000, A\&AS, 146, 323

Rameau, J., Chauvin, G., Lagrange, A.-M., et al. 2013, ApJ, 772, L15

Skemer, A. J., Hinz, P., Esposito, S., et al. 2014, in Adaptive Optics Systems IV, Proc. SPIE, 9148, 91480L

Soummer, R., Pueyo, L., \& Larkin, J. 2012, ApJ, 755, L28

Stolker, T., Bonse, M. J., Quanz, S. P., et al. 2019, A\&A, 621, A59

Takami, M., Bailey, J., \& Chrysostomou, A. 2003, A\&A, 397, 675

Tamura, M. 2014, in Exploring the Formation and Evolution of Planetary Systems, eds. M. Booth, B. C. Matthews, \& J. R. Graham, IAU Symp., 299, 12

Taylor, K. N. R., \& Storey, J. W. V. 1984, MNRAS, 209, 5P

Vigan, A., Patience, J., Marois, C., et al. 2012, A\&A, 544, A9

Wertz, O., Absil, O., Gómez González, C. A., et al. 2017, A\&A, 598, A83

Wilking, B. A., McCaughrean, M. J., Burton, M. G., et al. 1997, AJ, 114, 2029

Wolk, S. J., Rice, T. S., \& Aspin, C. A. 2013, AJ, 145, 113

Wright, E. L., Eisenhardt, P. R. M., Mainzer, A. K., et al. 2010, AJ, 140, 1868 